\title{
Neurophysiological, pharmacological and behavioral evidence for medial thalamic mediation of cocaine-induced dopaminergic analgesia
}

\author{
Bai C. Shyu ${ }^{1,4, *}$, Judith A. Kiritsy-Roy ${ }^{1,3,4}$, Thomas J. Morrow ${ }^{1,2,4}$ and \\ Kenneth L. Casey ${ }^{1,2,4}$ \\ Departments of ${ }^{1}$ Neurology and ${ }^{2}$ Physiology and ${ }^{3}$ Institute of Gerontology, University of Michigan, Ann Arbor, MI 48109 (U.S.A.) and \\ ${ }^{4}$ Neurology Research Laboratories, Veterans Affairs Medical Center, Ann Arbor, MI 48105 (U.S.A.)
}

(Accepted 8 October 1991)

Key words: Cocaine; Antinociception; Medial thalamus; D-2 Dopamine blocker; Morphine; Electrophysiological recording

\begin{abstract}
These studies examined the effects of cocaine on thalamic neurons that respond maximally either to noxious or to innocuous somatic stimulation. Cocaine attenuated high intensity electrically-evoked nociceptive responses of all 25 units studied in the parafascicular and central lateral nuclei of the medial thalamus. A dose of $1 \mathrm{mg} / \mathrm{kg}$ intraveneously (i.v.) suppressed medial thalamic unit discharge evoked by both noxious somatic stimulation $(49.4 \pm 8.7 \%$ of control response) and spinal cord stimulation $(76.2 \pm 6.6 \%$ of control response). The effect of cocaine on unit responses to noxious somatic stimulation was dose-related in the range of $0.3-3.5 \mathrm{mg} / \mathrm{kg}$ i.v. and was attenuated by eticlopride, a D-2 selective dopamine receptor antagonist. Morphine also suppressed noxious somatic evoked responses of medial thalamic units in a dose-dependent manner. Units in the lateral (ventrobasal) thalamus $(n=4)$ that responded only to innocuous stimuli were not affected by cocaine at doses up to $3.5 \mathrm{mg} / \mathrm{kg}$ i.v. Ibotenic acid lesions in the parafascicular nucleus of the medial thalamus attenuated the analgesic effect of cocaine in the formalin test. These results suggest that both cocaine and the parafascicular nucleus interact with dopaminergic mechanisms that attenuate nociceptive spinal projections to the medial thalamus.
\end{abstract}

\section{INTRODUCTION}

The activity of cocaine as a central analgesic has recently been described both in animal ${ }^{21}$ and human ${ }^{36}$ studies. In the rat formalin and hot plate tests, cocaine produces a dose-dependent antinociceptive response that is antagonized by D-1 or D-2 dopaminergic antagonists but not by doses of naloxone that reverse morphine analgesia. These data suggest that the antinociceptive effect of cocaine is mediated primarily by dopamine and is independent of endogenous opioid mechanisms of pain suppression. However, the central nervous system (CNS) sites and mechanisms mediating cocaine analgesia remain unclear.

Recent work in our laboratory demonstrates that a systemic dose of cocaine $(25 \mathrm{mg} / \mathrm{kg}$ intraperitonially, i.p.) that produces behavioral antinociception inhibits the nociceptive responses of neurons in the medial reticular formation and in the medial thalamus ${ }^{3,4}$. Nociceptive-responding neurons in both the reticular formation and the medial thalamus receive input from the dorsal horn via the spinothalamic (STT) or spinoreticular tracts $(\mathrm{SRT})^{7,16}$. Projections from the medial thalamus, particularly the parafascicular-centromedian (Pf-
CM) complex, to the midbrain periaqueductal gray (PAG) have been described ${ }^{23}$, and electrical stimulation of the medial thalamus produces pain relief in humans ${ }^{2}$, 31. A descending inhibitory system, mediated by monoaminergic neurons from the PAG to the spinal dorsal horn, is believed to play a critical role in pain modulation $^{13,14}$. Thus it appears that the medial thalamus, particularly the Pf-CM complex, is involved in activation of descending pain suppression mechanisms ${ }^{29}$. The antinociceptive effect of cocaine could result from a direct action within the medial thalamus and/or at the level of the spinal dorsal horn, or from an indirect activation of these descending supraspinal inhibitory mechanisms.

The present studies were designed to examine further the role of the medial thalamus, particularly the nucleus parafascicularis (Pf), in mediation of the antinociceptive action of cocaine. Neurophysiological experiments were used to demonstrate a dose-dependent, dopamine-mediated inhibitory effect of cocaine on responses of medial thalamic neurons to noxious somatic stimulation. In addition, behavioral testing procedures were used to ascertain whether cocaine analgesia requires the integrity of neuronal pathways originating in the Pf.

\footnotetext{
* Present address: Institute of Biomedical Sciences, Academia Sinica, Taipei 11529, Taiwan, The Republic of China.

Correspondence: J.A. Kiritsy-Roy, Veterans Affairs Medical Center, Research Service (151), 2215 Fuller Road, Ann Arbor, MI 48105, U.S.A.
} 


\section{MATERIALS AND METHODS}

\section{Electrophysiological experiments}

Surgical preparation. Male Sprague-Dawley rats (300-400 g b. wt.) were anesthetized with chloral hydrate $(400 \mathrm{mg} / \mathrm{kg}$ i.p. $)$. A tracheal tube was implanted, and a catheter was inserted into the tail vein. Rats were mounted in a stereotaxic head holder and spinal frame (David Kopf). A partial limited craniotomy was performed bilaterally over recording sites in the medial or lateral thalamus. In most experiments, a laminectomy was performed in the thoracic region $\left(T_{5}-T_{10}\right)$ and a lesion of the dorsal columns (about $T_{5}-T_{6}$ ) was made after the dura mater was removed.

Rats were paralyzed with succinylcholine $(2 \mathrm{mg} / \mathrm{kg}$ intraveneously, i.v.) and ventilated artificially. Rectal temperature was maintained at $37-38^{\circ} \mathrm{C}$ on a thermostatically-controlled heating pad. End tidal $p \mathrm{CO}_{2}$ was kept at approximately $3-4 \%$ by adjusting the end-tidal volume and the respiratory frequency. Supplemental doses of succinylcholine $(2 \mathrm{mg} / \mathrm{kg}$ i.v. $)$ and chloral hydrate $(100 \mathrm{mg} / \mathrm{kg}$ i.v.) were given at $30 \mathrm{~min}$ intervals or more frequently when recovery from paralysis revealed that flexion reflexes could be elicited.

Recording methods. Stainless steel microelectrodes (impedance $=$ $5-15 \mathrm{~m} \Omega$ at $100 \mathrm{~Hz}$ ), insulated except at the tip, were positioned stereotaxically in medial or lateral thalamus ${ }^{27}$ using a hydraulicdrive micromanipulator (David Kopf). Spontaneous and stimulusevoked potentials were amplified differentially to the indifferent electrode, positioned in the frontal sinus, and displayed on the oscilloscope. The extracellularly recorded action potentials of single neurons were converted by an amplitude window discriminator to standard logic pulses and fed to a minicomputer system (Data General) for on-line data processing or off-line data analysis.

Stimulation procedures. All units in the thalamus were selected for study on the basis of their response characteristics to natural stimulation of the peripheral receptive field (contralateral foot pad). In the medial thalamus, these units responded exclusively or maximally to noxious (pinching) stimulation with a forceps at intensities sufficient to produce aversive behavior in awake rats and pain in humans. Nociceptive responding units in the medial thalamus were activated by transcutaneous electrical stimulation in the receptive field using a pair of stainless steel needle electrodes inserted into the foot pad. Stimulus intensities of 5-10 mA $(0.2-2 \mathrm{~ms}$ duration), which have been shown to activate small diameter afferent nerve fibers (A- $\delta$ and $C$ ) in the rat $^{24}$, were used to drive the medial thalamic units. Units in the lateral thalamus responded only to non-noxious stimulation of the receptive field (brushing, stroking, tapping), and these cells were also driven by transcutaneous electrical stimulation in the contralateral footpad (1-10 mA, 0.1-0.2 ms duration).

Medial and lateral thalamic units that were activated by foot pad stimulation were also driven by electrical stimulation of the spinal cord. A pair of stainless steel electrodes (diameter about 50-100 $\mu \mathrm{m}$ ) separated by a distance of $2 \mathrm{~mm}$ and insulated except at the tip were inserted bilaterally into the exposed ventral lateral spinal cord white matter $\left(\mathrm{T}_{8-10}\right)$. The spinal cord and surrounding tissue were covered with paraffin oil. Constant current pulses (0.1-0.5 mA, 0.2 ms duration) were delivered via an electric stimulator (Grass, model S88) and stimulus isolation unit. Units activated by both footpad and spinal cord stimulation were similar in amplitude and waveform (Fig. 1), suggesting that the same neuron was being excited by both inputs. Fig. 1 displays the action potentials recorded from a single unit in Pf that was excited by electrical stimulation of the footpad and spinal cord.

Experimental protocol. Spontaneous activity and unit responses to peripheral and spinal cord stimulation were sampled before drug treatment. All drug treatments were administered as bolus injections through the tail vein catheter. In studies on the time course of cocaine effects, spinally- and peripherally-evoked responses of medial $(n=9)$ and lateral $(n=4)$ thalamic units were sampled 1, 5, 10,15 and $20 \mathrm{~min}$ following the injection of cocaine $(1 \mathrm{mg} / \mathrm{kg} \mathrm{i.v.}$.). Only one drug dosage was tested in each animal.
In subsequent studies ( $n=11$ ), cumulative dose-response curves for cocaine were obtained by administration of incremental doses $(0.1,0.2,0.4,0.8$ and $1.6 \mathrm{mg} / \mathrm{kg}$ i.v. $)$ at intervals of $90 \mathrm{~s}$. In 7 of these animals, a selective $\mathrm{D}-2$ dopamine receptor antagonist, eticlopride $(50 \mu \mathrm{g} / \mathrm{kg}$, i.v. $)$, was injected $10 \mathrm{~min}$ before administration of cumulative doses of cocaine $(0.1,0.2,0.4,0.8,1.6,3.2$ and 6.4 $\mathrm{mg} / \mathrm{kg}$ i.v.). In the remaining 4 rats, a control injection of saline was given. Spinally- and peripherally-evoked responses were sampled 60 $s$ after injection of each dose of cocaine.

Cumulative dose-response curves for morphine $(n=5)$ were obtained by administration of incremental doses of morphine $(0.09$, $0.19,0.38,0.75$ and $1.5 \mathrm{mg} / \mathrm{kg}$ i.v.) at $8 \mathrm{~min}$ intervals. Peripherallyevoked responses of medial thalamic units were recorded 4 min after each injection.

\section{Behavioral testing}

Lesioning technique. Rats $(400-500 \mathrm{~g})$ were anesthetized with - chloral hydrate $(400 \mathrm{mg} / \mathrm{kg}$ i.p.). Bilateral lesions of Pf $(n=9)$ were made with microinjections of $0.2 \mu \mathrm{l}$ of a solution $(10 \mu \mathrm{g} / \mu \mathrm{l})$ of ibotenic acid (IBO; Research Biochemicals Inc., Natick, MA) in phosphate buffered saline ( $\mathrm{pH} 7.5)$. Sham control rats $(n=11)$ received equivalent volumes of vehicle. Stereotaxic coordinates for Pf were A $-4.2 \mathrm{~mm}, \mathrm{~L} 0.3-0.4 \mathrm{~mm}$ and $6.5 \mathrm{~mm}$ down from the horizontal skull, according to the atlas of Paxinos and Watson ${ }^{27}$ Animals were allowed to recover at least 7 days before experiments.

Antinociceptive testing. The formalin test ${ }^{12}$ was used to determine the antinociceptive effect of cocaine in sham control and IBOlesioned rats. A solution of formalin $(5 \%, 0.05 \mathrm{ml})$ was injected subcutaneously into the plantar surface of one hindpaw. Beginning at $15 \mathrm{~min}$ after formalin injection, the animal's behavior was scored by an observer according to the number of seconds during a $1 \mathrm{~min}$ observation period that the animal exhibited the following: Grade 3 , paw fully elevated with constant licking or biting; Grade 2, paw fully elevated; Grade 1, partial weight bearing on the paw; Grade 0 , full weight bearing on the paw. Pain intensity ratings were calculated as the sum of the products of each grade and time spent in that grade divided by $60 \mathrm{~s}$. Three baseline pain scores were taken at $5 \mathrm{~min}$ intervals before injection of cocaine $(10-20 \mathrm{mg} / \mathrm{kg}$ i.p.), and scoring was continued up to $30 \mathrm{~min}$ after cocaine.

\section{Data analysis}

Post-stimulus discharge frequency (PSDF) was determined before and after drug treatments from computer-generated average peristimulus time histograms (PSTHs, 5-20 stimuli, $50 \mathrm{~ms}$ analysis period). Mean baseline PSDF was determined from 4-5 PSTHs obtained immediately prior to drug administration. The responses following drug treatments were compared with baseline PSDF and expressed as percent change from baseline. Responses from similarly treated animals were averaged, and data are routinely given as mean \pm S.E.M. discharge frequency (\% of pretreatment control). Treatment-related changes were considered significant $(P<0.05)$ as determined by the paired $t$-test. Differences between doses were assessed by ANOVA with Duncans Multiple Comparisons Test ${ }^{34}$.

In the behavioral studies, formalin test scores at each time point before and after cocaine treatments were averaged for lesioned and sham groups, and are given as mean \pm S.E.M. Between group comparisons were made using the Student's $t$-test ${ }^{34}$.

\section{Histology}

To identify the location of the evoked thalamic unit activity, a DC constant anodal current $(50 \mu \mathrm{A})$ was passed through the recording electrode for $30 \mathrm{~s}$ at the end of the experiment. A second lesion 1 $\mathrm{mm}$ dorsal to the first was made with a current intensity of $70 \mu \mathrm{A}$. The distance between these two lesion sites in the histological section was used to judge the extent of tissue shrinkage.

At the conclusion of all experiments, rats were perfused transcardially with normal saline followed by $10 \%$ formalin. Cryostat sections $(60-80 \mu \mathrm{m})$ of the brains were stained with Cresyl violet for identification of the recording sites or IBO lesions. Sections were 
examined under a light microscope and those with lesions of the Pf were also photographed. The area of the lesion was determined from the photomicrographs and charted onto representative drawings taken from the atlas of Paxinos and Watson ${ }^{27}$.

\section{RESULTS}

\section{Electrophysiological experiments}

A total of 29 thalamic units was studied. The distribution of the recording sites is shown in Fig. 2. The majority of units in the medial thalamus $(n=25)$ were scattered throughout the central lateral and Pf nuclei. The receptive fields of the medial thalamic units were identified using natural noxious stimulation (pinch), and all medial thalamic units studied responded either exclusively or with the maximum frequency and duration of discharge to noxious stimulation. Once identified in this way, medial thalamic cells were driven by transcutaneous electrical stimulation at intensities previously shown to activate small diameter afferents ${ }^{24}$. The mean latency of medial thalamic unit responses was $6.39 \pm 0.45 \mathrm{~ms}$ to electrical stimulation of the spinal cord and $13.23 \pm 0.68$ ms to high intensity electrical stimulation of the contralateral footpad.

Units $(n=4)$ in the lateral thalamus were located in the ventrobasal thalamus. All lateral thalamic units had receptive fields located in the contralateral hind limb and were maximally activated by natural innocuous stimulation. The lateral thalamic units were activated by electrical stimulation of the spinal cord and of the footpad. The mean latency of lateral thalamic unit responses was $5.17 \pm 0.31 \mathrm{~ms}$ and $8.67 \pm 1.33 \mathrm{~ms}$ with electrical spinal

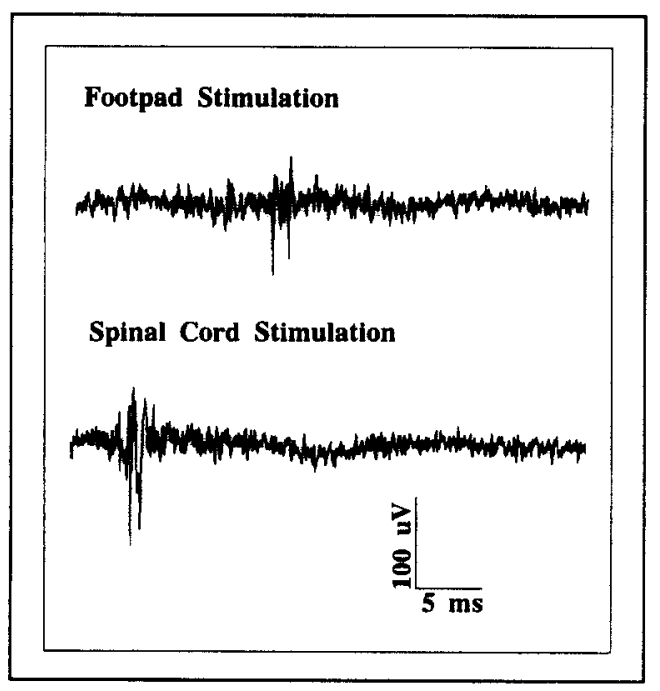

Fig. 1. Representative oscilloscope tracings of spike activity driven by electrical stimulation of the footpad ( $10 \mathrm{~mA}$, upper trace) and spinal cord $(500 \mu \mathrm{A}$, lower trace). Recording electrode was positioned in Pf of the medial thalamus. cord and foot pad stimulation, respectively. All units could be driven by both spinal cord and footpad stimulation.

Cocaine (1 mg/kg) suppressed the responses of medial thalamic units evoked by electrical stimulation of the contralateral foot pad (9/9) or spinal cord (7/9). An example of the effect of cocaine $(1 \mathrm{mg} / \mathrm{kg})$ is demonstrated in Fig. 3. Attenuation of the evoked responses by cocaine was not accompanied by any significant change in baseline spontaneous firing rate.

Fig. 4 demonstrates the time course of the suppressive effect of cocaine $(1 \mathrm{mg} / \mathrm{kg}$ i.v.) on medial thalamic unit responses to spinal cord and foot pad stimulation. The peak suppressive effect of cocaine $(49.4 \pm 8.7 \%$ of control) on thalamic responses to footpad stimuli occurred within the first minute, and the inhibitory effect of cocaine was apparent for the entire $20 \mathrm{~min}$ observation period. The response to spinal cord stimulation was significantly but less effectively suppressed $(76.2 \pm 6.6 \%$ of control), and only within the first $5 \mathrm{~min}$. Cocaine (1 $\mathrm{mg} / \mathrm{kg}$ ) had no effect on spinally or peripherally-evoked responses of lateral thalamic units (Fig. 5).

The effect of cocaine on medial thalamic unit responses

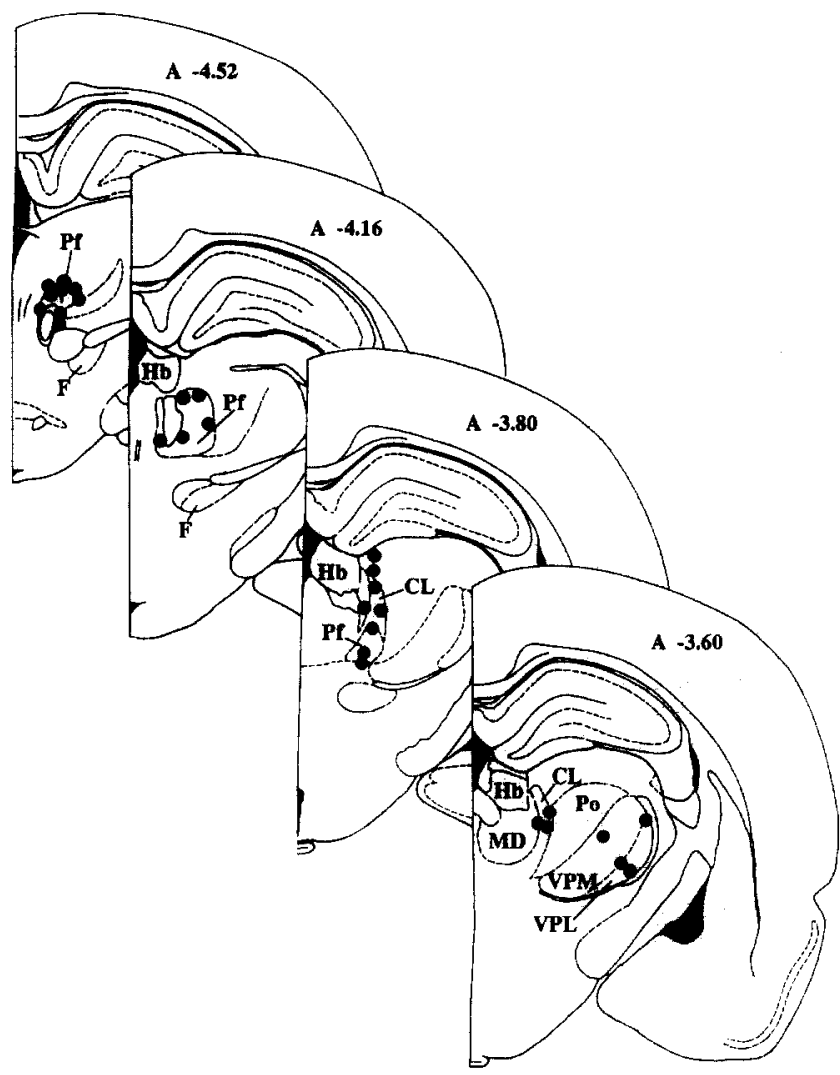

Fig. 2. Reconstruction of histologic sections depicting all recording sites within the medial and lateral thalamus. Sections were cut in the coronal plane, and the numbers represent the relative distance from bregma, according to the atlas of Paxinos and Watson ${ }^{27}$. 


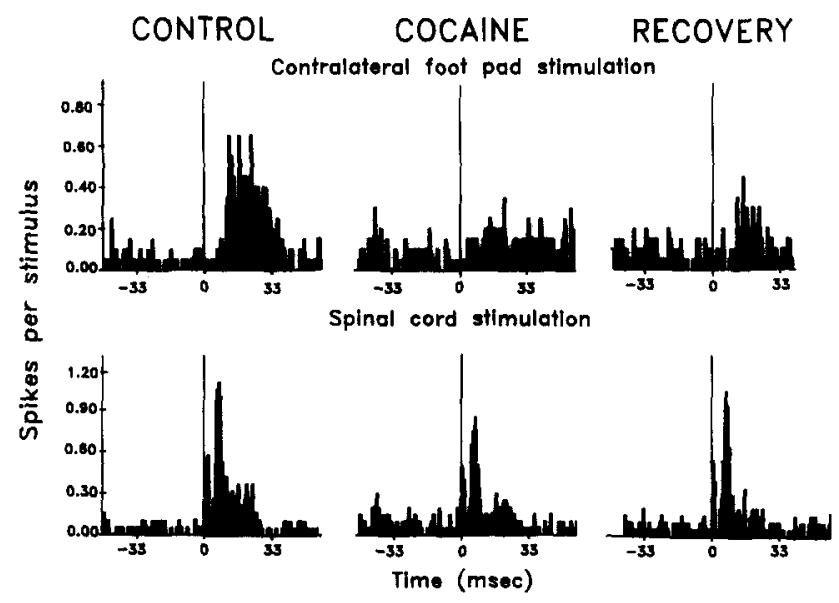

Fig. 3. Peri-stimulus time histograms showing the inhibitory effect of cocaine on the responses of one medial thalamic neuron evoked by contralateral footpad (upper panel) and spinal cord (lower panel) stimulation. Each histogram in this example represents the averaged response to 20 stimuli. The control histograms were obtained immediately prior to injection of cocaine $(1 \mathrm{mg} / \mathrm{kg}$ i.v. $)$. The histograms showing the inhibitory effect of cocaine were obtained at the time of peak cocaine effect (1-2 min after injection), and recovery histograms were taken at $20 \mathrm{~min}$ after injection of the drug. In this experiment, cocaine inhibited the response to electrical stimulation of the contralateral foot pad and spinal cord by $45.5 \%$ and $22.9 \%$, respectively.

to footpad stimulation was dose-related over the range of $0.1-3.5 \mathrm{mg} / \mathrm{kg}$ i.v. (Fig. 6). Administration of incremental doses of cocaine at $90 \mathrm{~s}$ intervals produced a cumulative dose-response curve with a maximal suppression of about $40 \%$ at the dose of $3.5 \mathrm{mg} / \mathrm{kg}$. Cocaine produced less suppression of spinally-evoked responses of the same medial thalamic units. Inhibition of about $5-10 \%$ was observed after the first dose $(0.1 \mathrm{mg} / \mathrm{kg}$ i.v. $)$ without

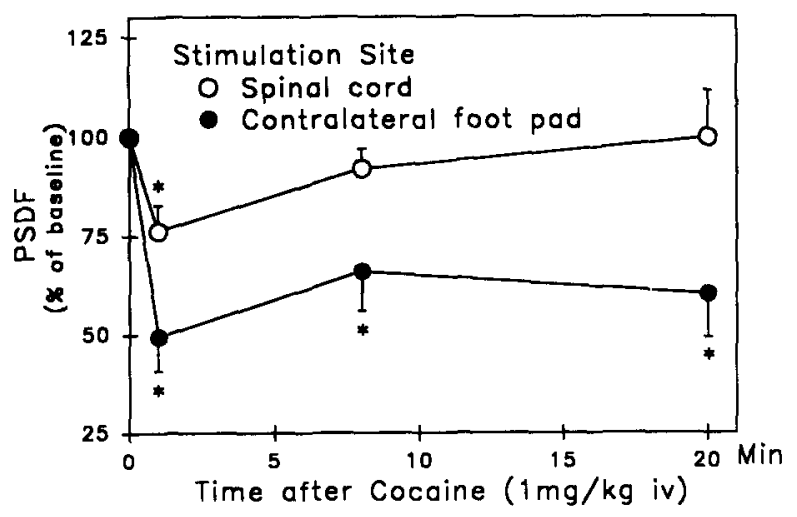

Fig. 4. Time course for the inhibitory effect of cocaine on medial thalamic unit responses to electrical stimulation of the spinal cord or contralateral footpad. Cocaine $(1 \mathrm{mg} / \mathrm{kg}$ i.v.) was injected at time $=0$. Each point represents the mean \pm S.E.M. evoked response, expressed as post-stimulus discharge frequency (PSDF) after cocaine as a percent of the pretreatment PSDF. An average of 4-5 control responses to each type of stimulus was obtained before cocaine injection. A total of 9 units was studied in this experiment. *Indicates a response significantly different from $100 \%$ of control at $P<0.05$ (paired $t$-test).

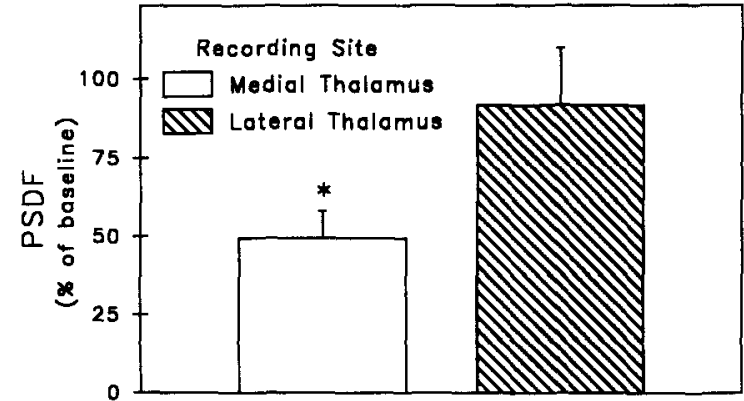

Fig. 5. Effect of cocaine (1 mg/kg i.v.) on peripheral somatic evoked responses of units in medial vs lateral thalamus. Responses are the mean \pm S.E.M. post-stimulus discharge frequency (PSDF) at $1 \mathrm{~min}$ after cocaine as a percent of the pretreatment PSDF for groups of medial $(n=9)$ and lateral $(n=4)$ thalamic units. ${ }^{*}$ Indicates a response significantly different from $100 \%$ of control at $P<0.05$.

further suppression at higher doses.

To determine if the effect of cocaine on medial thalamic unit responses to noxious somatic stimulation is mediated by a brain dopaminergic mechanism, a group of rats was pretreated $10 \mathrm{~min}$ before cocaine with eticlopride, a selective $D-2$ dopamine receptor antagonist. This treatment produced a shift to the right in the cumulative dose-response curve for cocaine-induced suppression of medial thalamic unit responses to footpad stimulation (Fig. 6).

For comparison, the effect of morphine, another centrally-active analgesic, on the responses of medial thalamic units to contralateral footpad stimulation was investigated. Cumulative doses of morphine, injected at

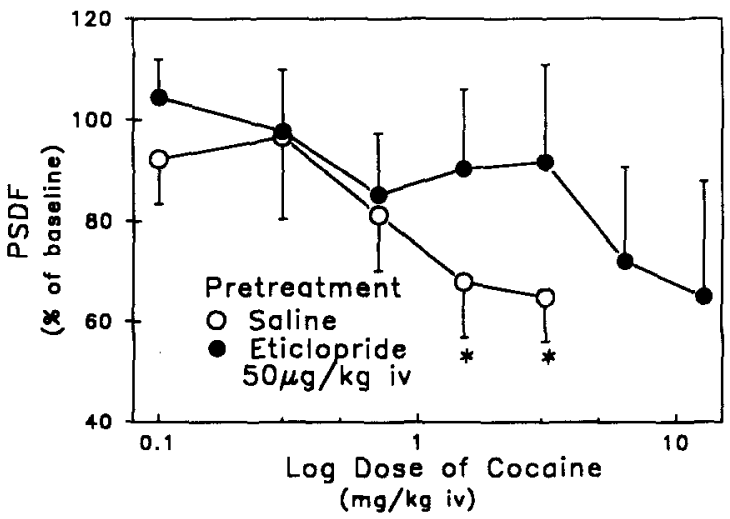

Fig. 6. Cumulative dose-response curves for cocaine following pretreatment with either saline $(n=4)$ or eticlopride $(n=7)$. Bolus injections of cocaine were given through the tail vein catheter at 90 $s$ intervals, and responses to the cumulative doses of $0.1,0.3,0.7$, 1.5 and $3.1 \mathrm{mg} / \mathrm{kg}$ were determined at 30-60 s after each injection. In studies with eticlopride pretreatment, additional doses of cocaine (6.3 and $12.7 \mathrm{mg} / \mathrm{kg}$ ), which were lethal in saline-pretreated animals, were injected in a similar manner without killing the rats. Responses, expressed as percent of the baseline post-stimulus discharge frequency (PSDF), are given as the mean \pm S.E.M. ${ }^{*}$ Indicates responses significantly different from $100 \%$ of control $(P<$ 0.05 as determined by the paired $t$-test). 


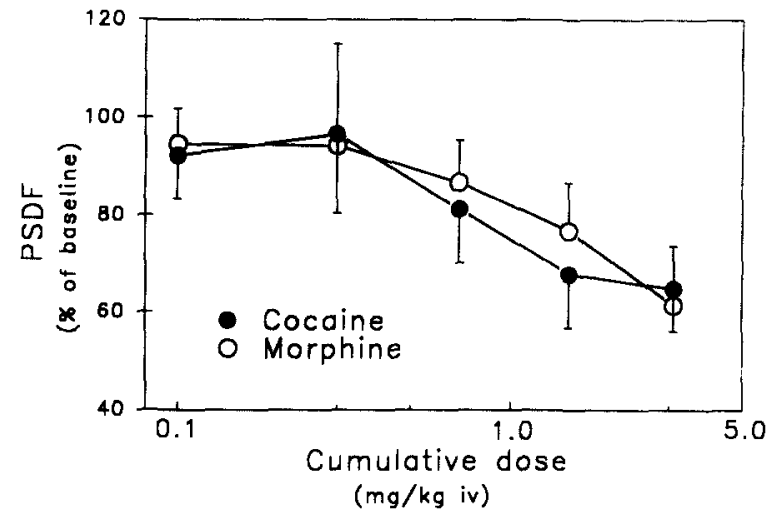

Fig. 7. Comparison of the cumulative dose-response curves for the effect of cocaine $(n=4)$ and morphine $(n=5)$ on noxious somatic evoked medial thalamic unit responses. Doses of cocaine and responses to each dose are those also shown in Fig. 5. Doses of morphine were given at $8 \mathrm{~min}$ intervals, and responses were determined at $4 \mathrm{~min}$ after each dose. Responses, expressed as percent of the average post-stimulus discharge frequency (PSDF) before drug injections, are given as the mean \pm S.E.M. ${ }^{*}$ Indicates responses significantly different from $100 \%$ of control $(P<0.05$ as determined by the paired $t$-test)

8 min intervals, suppressed medial thalamic unit responses to footpad stimulation in a dose-related manner that was quite similar to that produced by cocaine (Fig. 7).

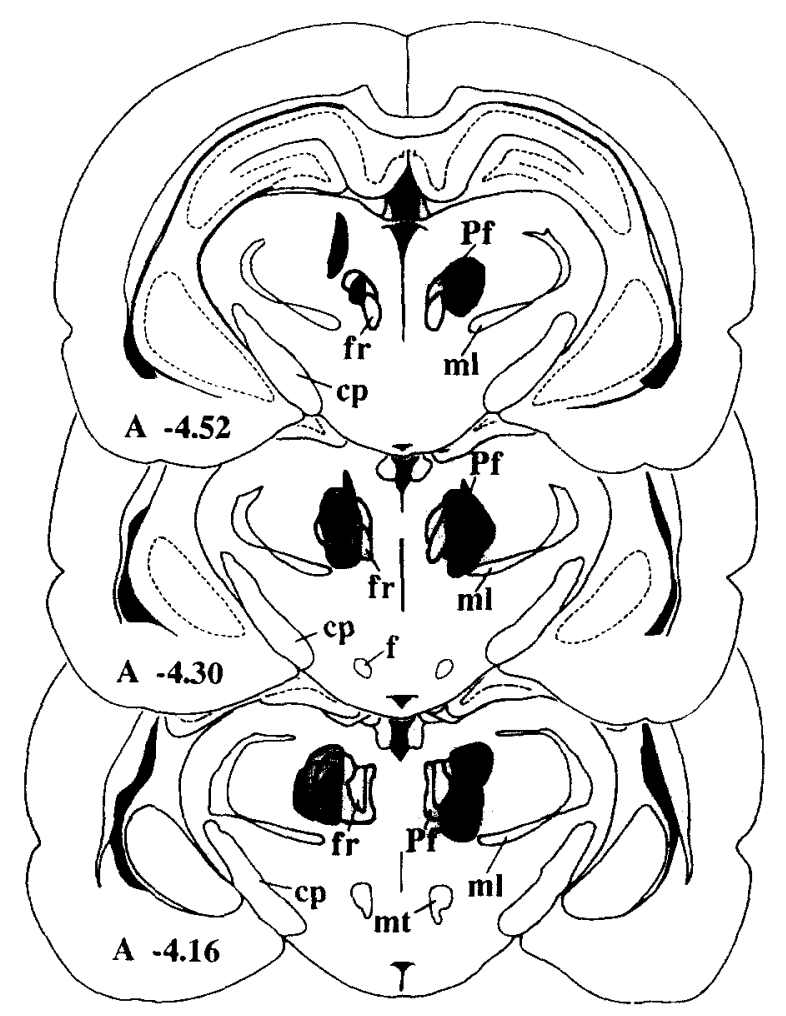

Fig. 8. Schematic representation of each of the IBO lesions in Pf. The areas affected by the neurotoxin in each of the 9 rats treated with IBO are outlined in frontal sections taken from the atlas of Paxinos and Watson ${ }^{27}$. The numbers in each section represent the distance (in $\mathrm{mm}$ ) from bregma.

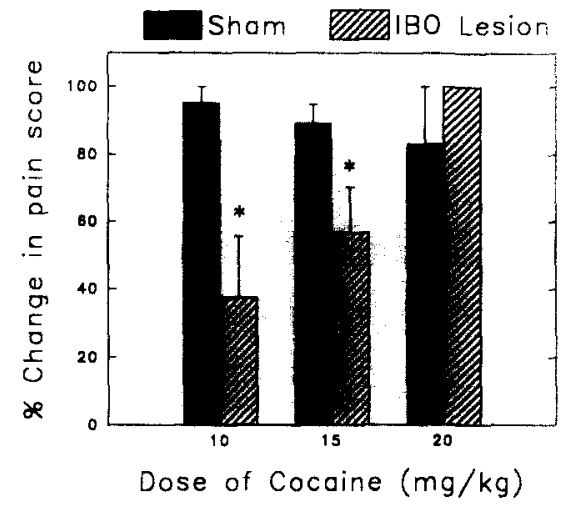

Fig. 9. Antinociceptive effect of cocaine in sham-operated control rats $(n=11)$ and in rats with bilateral IBO lesions of Pf $(n=9)$ There was no significant difference between sham and lesioned groups with respect to the average baseline pain score in the formalin test. Pain scores obtained at $30 \mathrm{~min}$ after injection of cocaine $(10-20 \mathrm{mg} / \mathrm{kg})$ in sham and lesioned groups are shown as \% change from baseline. ${ }^{*}$ Represents a significant difference between groups in pain score as determined by the Student's $t$-test $(P<0.05)$.

\section{Behavioral experiments}

Fig. 8 is a schematic representation of the extent of damage to Pf induced by microinjection of IBO in a group of 9 rats. In most cases, the treatment produced extensive damage to the neuronal population without significant damage to surrounding tissue or fibers of passage. Data from two additional animals with IBO injections outside of Pf were not included in the analysis. No damage to neurons in Pf was evident in histologic samples from rats $(n=11)$ treated with microinjections of vehicle in the area of Pf (sham control).

The formalin test was used to evaluate antinociceptive responses to several doses of cocaine in sham and IBO lesioned rats, and the results are shown in Fig. 9. At the highest dose $(20 \mathrm{mg} / \mathrm{kg}$ i.p. $)$, cocaine produced a robust antinociceptive response in both lesioned and sham groups, and the response was not significantly different between groups. However, with lower doses of cocaine, the antinociceptive response was significantly blunted in lesioned rats compared to sham controls. A dose of 10 $\mathrm{mg} / \mathrm{kg}$ of cocaine was clearly antinociceptive in sham rats, but was without effect in rats with IBO-induced lesions of Pf.

\section{DISCUSSION}

The results of these experiments suggest that the medial thalamus plays a key role in the mediation of the dopaminergic analgesia induced by cocaine. Cocaine analgesia is primarily dopamine-mediated and it is attenuated by lesions of the Pf. Systemic injections of cocaine suppressed medial thalamic responses to noxious peripheral somatic stimuli in a dose-related manner. The highest doses of cocaine inhibited the noxious evoked re- 
sponses of units in Pf and $\mathbf{n}$. centralis lateralis by about $50 \%$. This antinociceptive effect of cocaine could result from a direct inhibition of neurons in the medial thalamus, from a direct spinal inhibition of STT/SRT neurons, or by activation of descending inhibitory supraspinal pathways.

To examine the possible supraspinal mechanisms, we determined the inhibitory effect of cocaine on medial thalamic neuronal responses to spinal cord stimulation. Cocaine inhibited by about $25 \%$ responses of nociceptive units in the medial thalamus driven by electrical stimulation of the spinal cord, but this effect could not be shown to be dose dependent. Cocaine had a much greater effect on the somatically, as compared to the spinally, evoked unit responses. This differential effect could be due to a less synchronous, perhaps weaker, synaptic volley elicited by somatic stimulation. Additionally or alternatively, cocaine may affect both spinal dorsal horn and supraspinal synaptic transmission to medial thalamus. Because the dorsal columns rostral to the stimulation site were sectioned in all of the experiments using spinal stimulation, it is highly unlikely that the effect of cocaine is due to an action on neurons of the dorsal column nuclei or their rostral projections. Thoracic spinal cord stimulation could, via collateral fibers, activate synaptically dorsal horn neurons with ascending projections, but this would be unlikely to produce the unimodal, short latency responses we observed with spinal cord stimulation. The spinally-evoked responses of medial thalamic units were probably activated either directly by fibers ascending in the STT or indirectly via the SRT. Taken together, these results favor a supraspinal site for the effect of cocaine on spinally-evoked activity.

Dougherty et al. ${ }^{11}$ recently demonstrated that iontophoretic application of cocaine inhibits nociceptive responses of neurons in Pf, suggesting that at least part of the antinociceptive action of cocaine could be mediated directly at the Pf. To further investigate the role of the Pf in pain transmission and antinociception, we examined the effect of bilateral lesions of the Pf on baseline pain scores and on cocaine analgesia in the formalin test. Lesions were produced by microinjection of IBO, a neurotoxin that destroys selectively neuronal perikarya ${ }^{30}$. There was no difference between lesion and control groups with respect to the baseline pain score in the formalin, hot plate or tail flick tests. These findings agree with a previous report in which electrolytic lesions destroying the Pf had no effect on baseline responses in the rat tail flick, hot plate or shock titration tests ${ }^{35}$. Thus, neurons in this region are not essential for the perception of pain or coordination of the motor response to the noxious stimulus (formalin). Instead, it is thought that the Pf modulates the activity of descending pain suppres- sion systems. Reciprocal connections have been demonstrated between Pf and $P_{A G}{ }^{9,22,23}$, and stimulation of the PAG or dorsal raphe nuclei inhibits noxious responses of units in the $\mathrm{Pf}^{28}$. Electrical stimulation of $\mathbf{P f}$ and related medial thalamic areas provides pain relief in humans ${ }^{2,31}$.

The antinociceptive responses to cocaine at low doses of $10-15 \mathrm{mg} / \mathrm{kg}$ i.p. were attenuated in IBO-lesioned animals compared to sham-operated controls. This reduction in sensitivity to cocaine in Pf lesioned rats suggests that neurons in Pf mediate the antinociceptive effect of cocaine in the rat. However, additional sites and mechanisms appear to be involved, because a higher dose of cocaine $(25 \mathrm{mg} / \mathrm{kg}$ i.p.) produced similar antinociceptive responses in lesioned and control animals.

The effect of cocaine on medial thalamic nociceptive responses is antagonized by eticlopride, a D-2 dopamine receptor antagonist. Sulpiride, another D-2 dopamine receptor antagonist, blocked the inhibitory effect of iontophoretically-applied cocaine on nociceptive responses of neurons in $\mathbf{P f}^{11}$. In earlier studies, we found that the behavioral antinociceptive response to cocaine in the formalin and hot plate tests is antagonized also by eticlopride $^{21}$. Although cocaine inhibits neuronal reuptake of norepinephrine and serotonin as well as dopamine ${ }^{15,17,20}$, it appears that the antinociceptive effect of cocaine is mediated primarily through an effect on dopaminergic systems. Pf receives input from the A11 and A14 periventricular dopamine cell groups ${ }^{1}$. Dopaminergic neurons of the A11 area project also to the spinal cord, terminating in the superficial dorsal horn ${ }^{5,33}$. Fleetwood-Walker et al. ${ }^{14}$ demonstrated that focal electrical stimulation in the A11 region inhibited nociceptive responses of spinal dorsal horn neurons by dopaminergic mechanisms. Taken together, the results of the present experiments suggest that both cocaine and $P f$ neurons act through dopaminergic mechanisms at multiple levels of the neuraxis to inhibit noxious input. However, the role of the dopaminergic A11 cell group in mediation of cocaine analgesia at both spinal dorsal horn and medial thalamus will require further investigation.

Cocaine appears to inhibit specifically nociceptive input to the thalamus. Responses of low threshold neurons in the ventrobasal complex were not affected by any of the doses of cocaine used in these studies. This finding agrees with a previous study from our laboratory in which units in the ventrobasal complex of the lateral thalamus that responded only to innocuous somatic stimuli were unaffected by administration of systemic doses of cocaine that produce behavioral antinociceptive responses in the rat formalin and hot plate tests ${ }^{4}$. We did not study any nociceptive cells in the lateral thalamus, and therefore it is not known whether the effect of cocaine is specific for 
the medial thalamus or whether cocaine inhibits nociceptive input to all regions of the thalamus. If cocaine has an inhibitory effect on STT/SRT pathways at the spinal level, then it would suppress nociceptive input to lateral as well as medial thalamic neurons because the STT has collateral projections to both areas ${ }^{18}$.

We also found that morphine produced dose-related inhibition of medial thalamic unit responses to peripheral somatic nociceptive stimulation. This observation confirms previous reports suggesting that the medial thalamus plays a role in mediation of opioid analgesia ${ }^{6,8,10,28}$. However, it is unlikely that cocaine and morphine act by similar mechanisms to inhibit noxious input to the medial thalamus. Previous studies in our laboratory have demonstrated that doses of naloxone that inhibit the antinociceptive effect of morphine are without effect on cocaine analgesia in the hot plate or formalin tests ${ }^{21}$. Treatments that inhibit the behavioral antinociceptive effect of cocaine, such as eticlopride and medial thalamic lesions, have been shown to potentiate the same responses to morphine ${ }^{19,35}$. Cocaine has been shown to enhance the antinociceptive potency of opioid analge-

\section{REFERENCES}

1 Albanese, A., Altavista, M.C. and Rossi, P., Organization of central nervous system dopaminergic pathways, J. Neural Transm. Suppl., 22 (1986) 3-17.

2 Andy, O.J., Parafascicular-center median nuclei stimulation for intractable pain and dyskinesia (painful-dyskinesia), Appl. Neurophys., 43 (1980) 133-144.

3 Belczynski Jr., C.R., Pertovaara, A., Morrow, T.J. and Casey, K.L., The effect of systemic cocaine on the responses to noxious stimuli and spontaneous activity of medial bulboreticular neurons, Brain Research, 527 (1990) 204-212.

4 Belczynski Jr., C.R., Pertovaara, A., Morrow, T.J. and Casey, K.L., The effect of systemic cocaine on spontaneous and nociceptively evoked activity of neurons in the medial and lateral thalamus, Brain Research, 517 (1990) 344-346.

5 Björklund, A. and Skagerberg, G., Evidence for a major spinal cord projection from the diencephalic A11 dopamine cell group in the rat using transmitter-specific fluorescent retrograde tracing, Brain Research, 177 (1979) 170-175.

6 Carr, K.D. and Bak, T.H., Medial thalamic injection of opioid agonists: $\mu$-agonist increases while kappa-agonist decreases stimulus thresholds for pain and reward, Brain Research, 441 (1988) 173-184.

7 Casey, K.L. and Jones, E.G., An overview of ascending pathways: brainstem and thalamus, Neurosci. Res. Prog. Bull., 16 (1978) 103-118.

8 Cohen, S.R. and Melzack, R., Morphine injected into the habenula and dorsal posteromedial thalamus produces analgesia in the formalin test, Brain Research, 359 (1985) 131-139.

9 Comans, P.E. and Snow, P.J., Ascending projections to nucleus parafascicularis of the cat, Brain Research, 230 (1981) 337-341.

10 Dafny, N. and Gildenberg, P., Morphine effects on spontaneous, nociceptive, antinociceptive and sensory evoked responses of parafasciculus thalami units in morphine naive and morphine dependent rats, Brain Research, 323 (1984) 11-20.

11 Dougherty, P.M., Qiao, J.T., Wiggins, R.C. and Dafny, N., Microiontophoresis of cocaine, desipramine, sulpiride, methysergide and naloxone in habenula and parafasciculus, Exp. Neu- $\operatorname{sics}^{25,26,32}$, but the mechanism of this interaction and the role of the medial thalamus is not known.

In conclusion, the results reported here indicate that both cocaine and Pf neurons interact with dopaminergic mechanisms that inhibit noxious input to the medial thalamus. In producing dopaminergic analgesia, cocaine may act in parallel, rather than in series, with the Pf neurons because cocaine inhibits Pf neuronal nociceptive responses. The antinociceptive effect of cocaine is mediated by $\mathrm{D}-2$ dopamine receptors, suggesting that medial thalamic and spinal projections of dopaminergic neurons (possibly from the A11 cell group) are involved. An additional effect of cocaine directly at the spinal dorsal horn cannot be ruled out. Finally, the data provide further support for a role of the $\mathrm{Pf}$ in the regulation of descending pain suppression systems.

Acknowledgements. This work was supported by the Medical Research Service of the Department of Veterans Affairs and an award to K.L.C from Bristol-Myers Squibb. B.C.S. was supported by a Swedish Medical Consul Scholarship.

rol., 108 (1990) 241-246.

12 Dubuisson, D. and Dennis, S.G., The formalin test: a quantitative study of the analgesic effects of morphine, meperidine, and brain stem stimulation in rats and cats, Pain, 4 (1977) 161-174.

13 Fields, H.L. and Basbaum, A.I., Brainstem control of spinal pain-transmission neurons, Annu. Rev. Physiol, , 40 (1978) 217248.

14 Fleetwood-Walker, S.M., Hope, P.J. and Mitchell, R., Antinociceptive actions of descending dopaminergic tracts on cat and rat dorsal horn somatosensory neurones, J. Physiol., 399 (1988) 335-348.

15 Friedman, E., Gershon, S. and Rotrosen, J., Effects of acute cocaine treatment on the turnover of 5-hydroxytryptamine in the rat brain, Br. J. Pharmacol., 54 (1975) 61-64.

16 Giesler Jr., G.J., Menétrey, D. and Basbaum, A.I., Differential origins of spinothalamic tract projections to medial and lateral thalamus in the rat, J. Comp. Neurol., 184 (1979) 107-126.

17 Goeders, N. and Smith, J., Cortical dopaminergic involvement in cocaine reinforcement, Science, 221 (1983) 773-775.

18 Kevetter, G.A. and Willis, W.D., Spinothalamic cells in the rat lumbar cord with collaterals to the medullary reticular formation, Brain Research, 238 (1982) 181-185.

19 Kiritsy-Roy, J.A., Standish, S.M. and Terry, L.C., Dopamine D-1 and D-2 receptor antagonists potentiate analgesic and motor effects of morphine, Pharmacol. Biochem. Behav., 32 (1989) 717-721.

20 Langer, S. and Enero, M., The potentiation of responses to adrenergic nerve stimulation in the presence of cocaine: its relationship to the matabolic fate of released norepinephrine, $J$. Pharmacol. Exp. Ther., 191 (1974) 431-443.

21 Lin, Y., Morrow, T.J., Kiritsy-Roy, J.A., Terry, L.C. and Casey, K.L., Cocaine: evidence for supraspinal, dopaminemediated, non-opiate analgesia, Brain Research, 479 (1989) 306312.

22 Mantyh, P.W., Connections of midbrain periaqueductal gray in the monkey. I. Ascending efferent projections, J. Comp. Neurol., 206 (1982) 146-158.

23 Marchand, J.E. and Hagino, N., Afferents to the periaqueduc- 
tal gray in the rat. A horseradish peroxidase study, Neuroscience, 9 (1983) 95-106.

24 Menétrey, D., Geisler, G.J. and Besson, J.M., An analysis of response properties of spinal cord dorsal horn neurones to nonnoxious and noxious stimuli in the spinal rat, Exp. Brain Res., 27 (1977) 15-33.

25 Misra, A.L., Pontani, R.B. and Vadlamani, N.L., Stereospecific potentiation of opiate analgesia by cocaine: predominant role of noradrenaline, Pain, 28 (1987) 129-138.

26 Nott, M.W., Potentiation of morphine analgesia by cacaine in mice, Eur. J. Pharmacol., 5 (1968) 93-99.

27 Paxinos, G. and Watson, C., The Rat Brain in Stereotaxic Coordinates, Academic Press, New York, 1982.

28 Reyes-Vazquez, C., Qiao, J.-T. and Dafny, N., Nociceptive responses in nucleus parafascicularis thalami are modulated by dorsal raphe stimulation and microiontophoretic application of morphine and serotonin, Brain Res. Bull., 23 (1989) 405-411.

29 Sakata, S., Shima, F., Kato, M. and Fukui, M., Effects of thalamic parafascicular stimulation on the periaqueductal gray and adjacent reticular formation neurons. A possible contribution to pain control mechanisms, Brain Research, 451 (1988) 85-96.
30 Schwarcz, R., Hökfelt, T., Fuxe, K., Jonsson, G., Goldstein, $M$. and Terenius, L., Ibotenic acid-induced neuronal degeneration: a morphological and neurochemical study, Exp. Brain Res., 37 (1979) 199-216.

31 Shima, F., Aiko, Y., Hosokawa, S. and Kato, M., The analgesic mechanisms of the electrostimulation of the parafascicular nucleus, Neurosci. Res., Suppl. 1 (1985) 124S.

32 Shimada, A., Toshiharu, T. and Yanagita, T., Mode of potentiating action of cocaine in morphine analgesia, Jpn. J. Pharmacol., 48 (1988) 185-193.

33 Skagerberg, G. and Lindvall, O., Organization of diencephalic dopamine neurones projecting to the spinal cord in the rat, Brain Research, 342 (1985) 340-351.

34 Solal, R.R. and Rohlf, F.J., Biometry, W.H. Freeman, San Francisco, 1969.

35 Yaksh, T.L., Yeung, J.C. and Rudy, T.A., Medial thalamic lesions in the rat: effects on the nociceptive threshold and morphine antinociception, Neuropharmacology, 16 (1977) 107-114.

36 Yang, J.C., Clark, W.C., Dooley, J.C. and Mignogna, F.V. Effect of intranasal cocaine on experimental pain in man, Anesth. Anal., 61 (1982) 358-361. 